\title{
INFORME SOBRE COMERCIO INTERNACIONAL. ESTRATEGIAS EXPORTADORAS Y MEDIO AMBIENTE
}

\section{I.- Antecedentes.}

El Instituto de Estudios Internacionales de la Universidad de Chile ha llevado a cabo un proyecto conducente a la elaboración de un Documento de Trabajo sobre Comercio Internacional, Estrategias Exportadoras y Medio Ambiente, conforme al Contrato $\mathrm{N}^{\circ} 046-93$, suscrito con el Programa de las Naciones Unidas para el Desarrollo, PNUD, con fecha 3 de enero de 1994.

Para llevar a cabo este proyecto, el Instituto debía consultar la opinión de centros o académicos especialistas en materia ambiental, los cuales seleccionarian puntos específicos según una pauta común de trabajo. Los puntos que debían guiar la elaboración de los trabajos individuales de parte de los centros o académicos invitados eran los siguientes:

- Percepción general de la o las vinculaciones entre el desarrollo de la actividad empresarial y la protección ambiental;

- rol del Estado en la materia: a) instituciones y sus competencias; b) financiamiento y sus objetivos; c) el Estado como agente contaminante;

- aplicación del principio de "quien contamina paga" y posibilidad de reconversión;

- vinculación entre desarrollo científico y tecnológico y la aplicación de medidas ambientales a nivel de las empresas;

- medio ambiente y factores de competitividad;

- percepción de los temas globales: pérdida de la capa de ozono; cambio climático; biodiversidad. Incidencia en políticas de desarrollo a nivel productivo;

- recursos naturales, libertad económica y regulaciones ambientales.

Al mismo tiempo, el proyecto procuraba determinar de manera directa o indirecta, la existencia de conocimiento en nuestro país de la ciencia y la tecnología que permiten obviar los problemas que pudieran presentarse.

Junto a este propósito, se trataba de conocer acerca de los centros de excelencia que puedan apoyar en el futuro políticas o entregar solu- 
ciones específicas y precisar cuáles son las diferencias regionales que pudieran percibirse sobre el tratamiento o análisis del tema elegido.

Los centros invitados a participar fueron los siguientes:

- Centro de Economía de los Recursos Naturales y el Medio Ambiente, Facultad de Ciencias Económicas y Administrativas, Universidad de Chile.

- Centro de Estudios del Desarrollo.

- Centro de Estudios Públicos.

- Corporación Libertas.

- EADES-Georgetown.

- Libertad y Desarrollo.

- Universidad de Chile, Facultad de Ciencias Agrarias y Forestales.

- Pontificia Universidad Católica de Chile, Instituto de Estudios Urbanos.

Una vez elaborados los documentos individuales requeridos, se invitó a un taller de presentación de sus resultados y de debate, con el aporte de comentaristas seleccionados. Esta reunión tuvo lugar el dia 27 de abril de 1994, resultando en un productivo intercambio de opiniones de representantes del sector público, privado y académico.

Los análisis estuvieron dirigidos a determinar la relación que se plantea entre las exigencias ambientales, tanto sustantivas como institucionales, para una participación plena y crecientemente integrada del país en el actual esquema de relaciones económicas internacionales.

En este marco, se plantearon coincidencias fundamentales en los documentos elaborados en el marco del proyecto, en particular acerca del diagnóstico de la situación presente y las características que debe tener un enfoque global a nivel nacional acerca de cómo funciona el esquema de interrelaciones entre exigencias ambientales nacionales, regulaciones internacionales y perspectivas paralas economías exportadoras.

En este esquema, resultó importante constatar la coincidencia de intereses entre ramas de sectores productivos, como el forestal y de la industria de la madera, con la profundización de un rol más activo en la promoción pública privada de exportaciones ambientalmente certificadas o sustentadas, tarea común para el sector público y el privado.

Existe acuerdo acerca de que la prioridad que se otorga a la temática ambiental está vinculada a la conciencia de la sociedad frente a estos temas, la cual es incipiente en Chile. Se constata un esfuerzo de búsqueda de enfoques políticos, económicos y jurídicos que permitan abor- 
darla de manera eficaz, en el marco de una política económica de mercado.

El Programa de las Naciones Unidas para el Medio Ambiente (PNUMA) ha destacado que el medio ambiente comprende un sistema global complejo, de múltiples y variadas funciones y con una cantidad innumerable de interrelaciones en un proceso dinámico y evolutivo, integrado por el conjunto de los sistemas físicos, biológico, social, económico, político y cultural en que viven el hombre y los demás organismos.

En una perspectiva general, puede resultar exagerado proclamar la vigencia de una "economía ecológica de mercado", pero tiene considerable fundamento la propuesta de reforzar la dimensión ecológica dentro de un marco económico fundado principalmente en la asignación de recursos por la vía del mercado. El sistema económico de planificación central ha sido en la práctica de peores resultados que otros, en términos de impacto ambiental.

En Chile, se observa una naciente conciencia politica ambiental, aunque el tema seguiría siendo un fenómeno secundario en relación con otros más atractivos de la agenda nacional. Es un asunto más bien "reactivo", lo que tiene que ver con la estacionalidad de las crisis y las actitudes del público. En los sectores políticos existen amplias brechas entre las creencias que se expresan y las conductas cotidianas.

Los chilenos son víctimas de la "paradoja de la agregación", que consiste en que cada individuo cree que su propia conducta agrega muy marginalmente a las dimensiones del problema ambiental, y que por otra parte una conducta más responsable mejoraría las cosas en un grado tan imperceptible, que no resulta racional en la práctica elegir el camino del sacrificio de una cuota de comodidad y egoísmo.

Ello contrasta con los patrones culturales imperantes en los paíss más desarrollados donde las obligaciones de todos vinculan a cada uno como persona. Una clave del progreso en materia ambiental en nuestro país puede estar en lograr poner en marcha un cambio cultural profundo que nos permita salir de la trampa señalada y que parece conllevar una inexorable degradación ambiental.

Este razonamiento implica tener en cuenta la dimensión económica de la política ambiental. Una conducta propiamente ecológica debe ser rentable tanto para el consumidor como para el productor, lo que permitirá acceder a las ventajas del mercado a una actividad económica sostenida, ahorrativa de recursos y compatible con el medio ambiente. Es necesario propiciar más la economía de mercado en la protección dell medio ambiente. Es una de las conclusiones que comparten diferentes 
I.E.I. / Informe sobre comercio internacional, ...

trabajos realizados, en lo que respecta a los sectores productivos orientados a la exporțación.

Por otra parte, el concepto de que "el que contamina paga" involucra una responsabilidad esencial para lograr la internalización de los costos ambientales en el sistema de precios. De esta manera, puede darse una conducta ecológicamente responsable, de manera racional y rentable, $y$ : hacer justicia en la competencia entre los diversos agentes que operan en el mercado.

Tal responsabilidad, a su vez, generaría nueva actividad económica al estimular la demanda por bienes y servicios de saneamiento ambiental que incorporan tecnología no contaminante o descontaminante. En la medida en que determinados agentes económicos sobrepasen las exigencias de las normas de emisión, surge la posibilidad de crear un mercado de permisos transables de emisión, el que sin aumentar los nïveles totales de contaminación, puede ofrecer soluciones transitorias por la vía de la compra de permisos a aquellos agentes económicos que aún no logran satisfacer las exigencias ambientales fijadas por la autoridad.

Pero para que todo ello funcione realmente, es indispensable contar con una organización administrativa en lo ambiental que cumpla eficazmente las tareas de reglamentación y fiscalización. El desarrollo de esta capacidad administrativa permite operar los mecanismos de mercado destinados a resguardar el medio ambiente.

Finalmente, la interdependencia entre las políticas ambientales internas y externas es evidente y se hace sentir en las demandas de protección del medio ambiente que emanan tanto de los principales socios comerciales de Chile como de los inversionistas extranjeros que el pais se esfuerza en atraer.

Este punto será examinado en la siguiente sección de este Informe:

\section{II.- Principales conclusiones acerca de la relación entre comercio y desarrollo.}

\section{La determinación de los intereses de Chile en un escenario internacional de regulaciones ambientales.}

En relación a los requerimientos medio ambientales y el comercio internacional, se distinguen distintas áreas, que comprenden tanto los productos mismos como los procesos de producción, en particular: 
- las medidas comerciales, que tienen como objetivo controlar el ingreso de ciertos productos por razones medioambientales;

- las regulaciones medioambientales, que incluyen estándares implementados en los países industrializados sobre productos y prócesos de producción para proteger al medio ambiente y la salud pública, como las referidas a embalaje y etiquetado y que son regulaciones obligatorias que hay que cumplir;

- las políticas e instrumentos medioambientales, categoría que se refiere a los instrumentos económicos que ejercen impactos indirectos sobre los mercàdos internacionales a través de cambios en los precios nacionales así como en la demanda y oferta doméstica, como los subsidios, impuestos y cuotas de producción, que han tenido un impacto significativo sobre el comercio internacional. Estas jugarán un rol más y más importante en las políticas ambientales en el futuro; $y$,

- las conductas ecológicas o mercados verdes, que tienen relación con las transformaciones de los intereses de los consumidores y la emergencia de sistemas voluntarios establecidos por el sector privado para satisfacerlos y estäblecer la diferenciación de productos que se producen bajo tecnologías ambientalmente más favorables. Estos mercados verdes puéden significar oportunidades o barreras para el comercio. El "Angel Azul" de Alemania, el "Nordic Swan" de los países escandinavos y el "Green Cross" o "Green'Seal" de Estados Unidos, certifican la mejor calidad ambiental del pröducto que lleva el distintivo. Se nota, sin embargo, uná incipiente saturación del mercado por el uso indiscriminado de estos sellos.

La Comunidad Europea ha llegado a una "segunda generación" de los instrumentos medioambientales, caracterizada por un énfasis en instrumentos basados en el mercadó donde se radicará la gestión ambiental y que se ejercen dentro dé là èmpresa, enfatizando la prevención. Se trata de una gestión ambiental integral, denominada "life cycle analysis". Se introduce el sistema de "Ecoaudito" que prescribe el manejo integral de la gestión ambiental a nivei de la empresa, incluyendo la creación de balances ambientales y declaraciones públicas periódicas acerca de los problemas ambientales que enfrenta cada empresa.

Ante este escenario, el diseño de una política nacional respecto de las regulaciones medioambientales que imponen otros países, debe tener en cuenta ciertos aspectos relevantes:

a) La compatibilidad de las exigencias del mercado externo y del mercado interno. Las regulaciones medioambientales de los países in- 
dustrializados pueden ejercer impactos significativos sobre las exportaciones de los países en desarrollo y, en muchos casos, a éstos no los queda otra alternativa que integrar esas regulaciones en el ambiente industrial doméstico.

El rol del Gobierno consiste en percibir cambios en la opinión de la ciudadanía acerca del medio ambiente y transmitir estos cambios sin demora a la legislación e institucionalidad ambiental en el país y, por otra parte; debe procurar la minimización de las diferencias que existen en las regulaciones medioambientales en los países industrializados respecto de las existentes en Chile.

b) La compatibilidad de estudios de impacto ambiental. En esta materia existe una brecha considerable entre los países industrializados y Chile.

La "segunda generación" de políticas ambientales implica tres cambios:

- Desde políticas específicas hacia una política dentro de un marco general más bien orientador;

- desde el control estatal hacia un manejo de la gestión ambiental dentro de la empresa; y,

- desde un enfoque de impactos ambientales individuales hacia un enfoque de impactos ambientales totales e integrales, como el Ecoaudito, que incluye no solamente una descripción detallada de todas las actividades productivas y sus consecuencias sobre el medio ambiente, sino también una presentación de la política y del programa ambiental de la empresa.

Si el sistema del Ecoaudito se convierte en rutinario en läs empresas de los países industrializados y la evaluación del impacto ambiental de los productos se presenta como exigencia relacionada con las actividades productivas, será imprescindible que la industria chilena tome en cuenta estas tendencias.

c) La integración en el mercado internacional. A través de la participación activa en la negociación y el diseño de los convenios intermacionales, el Gobierno podrá transmitir una imagen positiva en relación a la protección del medio ambiente.

En lo relativo al Tratado de Libre Comercio de América del Norte (NAFTA), el tema del medio ambiente jugará un rol predominante. Chile puede aprovechar $y$ aprender de la experiencia de las negociaciones entre Estados Unidos y México para preparar una estrategia para las negociaciones pendientes en relación a su incorporación, teniendo como objetivo una creciente liberalización del comercio internacional 
y fortaleciendo al mismo tiempo las bases fundamentales de la protección ambiental.

Debe analizarse, por una parte, ỉa compatibilidad entre un comercio liberalizado y la protección ambiental $y$, por otra, la compatibilidad entre las legislaciones ambientales vigentes para los distintos sectores industriales en los países contratantes. La institucionalidad y el instrumental normativo medioambiental en Chile aún es insuficiente frente a un posible tratado de libre comercio con Estados Unidos, a pesar de la Ley de Bases del Medio Ambiente. Debe diseñarse una estrategia global económica ambiental y otra de negociación propiamente tal, lo que deberá hacerse a la brevedad.

\section{Aspectos especificos que surgen en la relación entre comercio internacional y medio ambiente.}

Algunos estudios directamente relacionados con el comercio internacional y la ecuación medio ambiente recursos naturales, han buscado evaluar los efectos esperados de un acuerdo de libre comercio entre Chile y Estados Unidos en los sectores agropecuario y agroindustrial chilenos, teniendo en cuenta los flujos de comercio y el empleo en los sectores agropecuario y agroindustrial.

Igualmente, se ha estudiado los efectos sobre el empleo de un tratado de libre comercio entre Chile-Estados Unidos: el caso de los sectores agroindustrial y agropecuario chilenos. Este tema aparece como central en una eventual negociación, en una dimensión comparable a la ambiental en su importancia. Los efectos parecerían desagregarse por regiones, aspecto que también es de considerar en materia ambiental.

En lo que se refiere a los alcances y perspectivas de las políticas ambientales como barreras proteccionistas en el comercio internacional, uno de los puntos de especial interés para Chile es el impacto que tiene la legislación de la Comunidad Europea o de sus países miembros sobre las exportaciones chilenas. Como un ejemplo concreto, se señala la Ley Topfer (Alemania) que afecta a las exportaciones chilenas, con un efecto equivalente a un arancel $a d$-valorem, junto a otras legislaciones de reciclaje europeas que comienzan a emerger y que afectan negativamente la competitividad de las exportaciones.

Para enfrentar esta situación, se recomienda la coordinación con otros exportadores, procurando crear mayor capacidad de negociación y aprovechar economías de escala en la reestructuración tecnológica re- 


\section{I.E.I. / Informe sobre comercio internacional,}

querida. Las medidas sanitarias y fitosanitarias de la CEE tämbién pueden generar una reducción en el comercio ante la eventual imposibilidad de alcanzar los estándares establècidos, así como por los mayores costos que ellos implican. La Tegisslación sanitaria posee un alto grado de ambigüedad y disćrecionalidad que permite su uso abusivo tanto en la fase de evaluación de riesgos como en la selección y aplicación de las medidas sanitarias đé gestión de riesgo.

La Ley Topfer habría âfectado a las empresas chilenas al aumentar los costos incurridos por los productores de envases y embalajes y por los exportadores y productores chilenos. Esta ley obliga a fabricantes y distribuidores que venden en Alemania a retomar los envases y embalajes para reutilizarlos o reciclarlos en forma paralela e independiente del sistema público de eliminación de residuos.

En esta materia, se advierte la urgencia de que funcione un mecanismo ágil de coordinación entre sector público y privado, que facilite la adaptación a las exigencias externas, la adecuación tecnológica y la incorporación en los costos correspondientes.

En materia de vinculación entre comercio internacional y libre comercio y sus implicancias para el sector laboral y ambiental en Chile, los estudios reflejan la percepción de que es la relación con Estados Unidos (o América del Norte) la que influye en mayor medida en materias como el empleo y el medio ambiente, lo cual podría no ser necesariamente cierto en la práctica y ser más exigente la situación europea.

Los efectos sobre èl mercado laboral serían expansivos en èl largo plazo, señalan algunas conclusiones, ya que a corto plazo se generaría la contracción de algunos sectores. Para paliar los efectos negativos sería fundamental adoptar medidas que incrementaran el nivel del capital humano y la productividad de la mano de obra, y expandir los sectores económicos relacionados con la investigación, el desarrollo y el progreso tecnológico.

Por otra parte, habría una expansión de la demanda por productos intensivos en recursos naturales, lo cual debería generar un marco regulatorio que garantice su uso eficiente en el largo plazo.. Esto implicaría una mayor "disciplina ambiental" producto del establecimiento de estrictos estándares ambientales sobre nuestras exportaciones.

Concluyendo acerca de estos puntos que vinculan las relaciones internacionales comerciales en un sentido amplio y la preocupación ambiental, las conclusiones concuerdan en señalar que Chile es un país en desarrollo, con un crecimiento sustentado en exportaciones estrechamente vinculadas a los recursos naturales. En este escenario, existe una 
relación positiva entre aperturas comerciales y posibilidad de abordar seriamente desde los ángulos más diversos la protección ambiental, teniendo siempre como horizontè el objetivo final de eliminación de la pobreza, acentuada por la falta de desarrollo y gran depredadora del medio ambiente.

De otro lado, la inserción internacional de Chile en materias comerciales/ambientales, no es un simple ejercicio de armonización de legislaciones o estándares ambientales. Se debe proponer establecer normas objetivas mínimas, como una etapa previa a la:aplicación automática de medidas unilaterales de países desarrollados, como parte de instrumentos más penalizadores que promotores de cambios de las conductas ambientales.

Se propone que las regulaciones ambientales a nivel internacional tengan las siguientes características:

- En cuanto al alcance: las políticas nacionales ambientales no deben proyectarse y trasladarse a países que poseen condiciones distintas;

- en cuanto a su necesidad: las políticas deben, ser empleadas como último recurso para corregir problemas ambientales y no como medidas distorsionadoras del comercio;

- en cuanto a la discriminación: debe evitarse la protección disfrazada.

En último término, este esquema debe ir acompañado de un sistema adecuado de solución de controversias.

\section{III.-EI medio ambiente desde la perspectiva de sectores específicos. La respuesta a las exigencias ambientales externas y las necesidades de políticas específicas.}

\section{El sector forestal.}

La industria forestal chilena ha crecido significativamente en los últimas décadas, lo que ha ido acompañado de constantes debates acerca del impacto de dicho proceso sobre los bosques nativos. Se percibe que la plantación de Pino Radiata y otras especies exóticas son una amenaza para el bosque nativo. También se considera una amenaza la explotación de esos bosques para la producción y exportación de astillas. La disminución del bosque nativo acarrea peligros asociados a la erosión, a la pérdida de diversidad biogenética y otros. 
En este sentido, los estudios preparados en el marco del proyecto concluyen que deben elaborarse objetivos para el sistema nacional forestal como un todo. Los principales roles del recurso forestal en Chile serían el de preservación, protección y producción.

El rol de preservación se refiere a la mantención de la biodiversidad de especies de flora y fauna, lo cual genera diversos beneficios, como asegurar el equilibrio ecológico en los ecosistemas y mantener una reserva genética para el futuro y permite a la población disfrutar de la belleza escénica de un ecosistema adecuadamente preservado.

En cuanto a la protección de suelos, los bosques reducen la variabilidad de los caudales a través de una mayor retención de agua del subsuelo apoyando así labores productivas como la agricultura, factor especialmente importante si se considera la montañosa geografia del país.

El rol de la producción consiste en contribuir de modo sustentable al desarrollo general del país por la vía de la generación de valor agregado con sus implicancias directas.

El diagnóstico de la situación actual en cuanto a la preservación indica que el rol de la preservación es llevado a cabo íntegramente por el SNASPE (Sistema Nacional de Areas Protegidas) y se detectan problemas de gestión, como la existencia de una inadecuada representatividad de todos los ecosistemas del país, la ausencia de parques nacionales cerca de los centros más densamente poblados y la falta de productividad de ciertos bosques que aportaría mayores recursos para que el SNASPE cumpla cabalmente con su función.

Respecto del rol de protección, existen alrededor de 12 millones de hectáreas en las zonas con aptitud forestal que corresponderian a suelos de protección en manos del sector privado. En lo que se refiere a la producción, más del $90 \%$ del valor de la producción formal del sector forestal es aportada por las plantaciones de crecimiento rápido constituidas por especies exóticas.

La contribución actual del sector forestal a la economía del pais se podría multiplicar entre 3 y 4 veces de aquí al año 2000, logrando para esa fecha un nivel de producción constante que no afecte el stock de masa boscosa y sin afectar la función protectora. Se concluye que el objetivo general de la legislación debería ser permitir el pleno aprovechamiento del potencial productivo del país sujeto a la restricción que se sostenga y aumente la protección a los suelos frágiles y cuidando que se satisfaga el objetivo de preservación que el país como un todo está dispuesto a satisfacer.

El Proyecto de Ley de Bosque Nativo, marco que regularía esta materia, es criticado por cuanto los instrumentos propuestos para alcan- 
zar los objetivos de "... lograr la conservación y uso racional de las formaciones vegetales naturales, especialmente de los bosques nativos...", no se estiman adecuados.

Por un lado se considera la utilización de incentivos económicos, y por otra parte se contempla la imposición de prohibiciones y obligaciones para los propietarios de bosque nativo dependiendo del tipo de bosque en cuestión. La combinación de incentivos y restricciones lleva a algunas contradicciones de importancia.

Las prohibiciones, si bien pueden tener efectos positivos en términos de preservación, eliminan los incentivos provistos para aumentar el bosque nativo precisamente en aquellos casos en que éste se valoraria más. Además, el proyecto de ley provee un marco muy estrecho de alternativas para la acción privada y, por otra parte, contiene cláusulas dificiles de justificar como aquélla que exime de la obligación de reforestar con especies idénticas a las originales en el caso en que se recupere el terreno para fines agrícolas. La implementación, fiscalización y control de esta ley recaerá básicamente en la Corporación Nacional Forestal (CONAF), lo que implica una gran carga de responsabilidad.

Se proponen algunos aspectos claves para una política forestal, como proporcionar los incentivos apropiados al sector privado de forma de lograr preservar y ojalá aumentar los tipos de bosques que serían más valorados por la sociedad. En cuanto a la preservación, el SNASPE debe lograr una adecuada representatividad de los ecosistemas del país, debe crear parques nacionales más cerca de los centros más poblados $y$, debe integrar a otros agentes a estas labores como por ejemplos fundaciones y corporaciones privadas nacionales asi como organismos internacionales. Adicionalmente, se propone la creación de un Fondo para la Preservación, con aportes de CONAF, de empresas privadas y de otros organismos nacionales e internacionales.

En lo que se refiere a la protección, se propone la creación de un subsidio para protección de suelos frágiles mediante la forestación de dichos suelos; y respecto a la producción, se propone permitir la explotación forestal en suelos que no sean frágiles, sin considerar subsidios de costos y planes de manejo flexibles para aprovechar la capacidad creativa del sector privado. 


\section{Las maderas provenientes del bosque nativo.}

Su relación con la biodiversidad.

a) Características de la situación vigente:

Las exigencias para los productos forestales pretenden que la madera sea obtenida con criterios de sustentabilidad de los bosques en el país de origen y, que el producto sea compatible con el medio ambiente y la salud humana en el país de destino. Esto se debe a la alarmante eliminación de bosques naturales en el mundo, junto al fenómenó social que concede mayor interés a la mantención de los bosques por su valor ecológico que por su valor comercial.

A nivel internacional, hay consenso y criterios generales respecto a que la madera debe proceder de explotaciones sustentables. En la práctica, el problema es complejo y de dificil solución. Sin embargo, los gobiernos se verán obligados a tomár medidas en el corto plazo, presionados por la gravedad del problema y por la importancia política del tema en la opinión pública.

Los requerimientos de compatibilidad ambiental del producto son simples, precisos y efectivos. Las exigencias ambientales son razonables, no excluyen el uso forestal de los bosques naturales y a la larga benefician directamente a los países productores. Sólo obligan a realizar la producción forestal con criterios técnicos de mantención del recurso y a una utilización sin deterioro ambiental El problema consiste en que una producción sustentable con métodos ambientalmente compatibles es más cara que la forma tradicional de explotación.

La destrucción de bosques naturales en el mundo, especialmente la eliminación de las șelvas tropicales, es una de las grandes preocupaciones en el debate ambiental. Sin embargo, la mayor parte de la madera industrial que se utiliza en el mundo proviene de plantaciones artificiales. En los paises en desarrollo, los bosques naturales son más importantes como proveedores de combustibles.

En el concierto mundial, la participación de Chile es baja; sin embargo a nivel del país, el sector forestal es uno de los sectores importantes, más dinámicos y con una evolución favorable. No obstante, la información estadística en lo que a bosques nativos se refiere es precaria, inexacta y obsoleta.

El estado actual de cobertura forestal y el uso de los suelos forestales reflejan un grave deterioro causado por la destrucción y transformación de los bosques nativos para la agricultura y la ganadería, y en menor grado por la explotación maderera. El desarrollo forestal se sustenta 
fundamentalmente en la producción silvícola de las plantaciones, que se han establecido mayoritariamente en suelos degradados por actividades agropecuarias. Se planta más y se consume menos de la mitad del crecimiento de los bosques.

Esto no es así en el caso de los bosques naturales, los que están sometidos mayoritariamente a una explotación selectiva (floreo), sin manejo silvícola. El ritmo de destrucción se ha mantenido, pero el manejo propiamente tal no es adecuado. Se concluye que Chile está distante de aplicar la técnica silvícola y utilizar satisfactoriamente sus bosques naturales, pero existirian las condiciones de mercado, la voluntad política y la capacidad técnica y empresarial para orientar correctamente el uso forestal con un sentido ecológico, económico y social.

Desde el punto de vista productivo, los bosques nativos son de baja calidad. La cantidad y calidad de productos aprovechables y su crecimiento son muy inferiores a los potenciales con silvicultura. En el bosque nativo chileno hay un gran potencial, lo que lo sitúa favorablemente entre los buenos bosques productivos del mundo. Pero estos rendimientos y sobre todo el mejoramiento cualitativo sólo son posibles con intervenciones silviculturales (serie de intervenciones productivas y no productivas a lo largo de la rotación del bosque) desde el inicio.

En el bosque manejado se llega a un nuevo equilibrio en que la mayoría de los aspectos ambientales del bosque natural pueden mantenerse y no son afectados negativamente. El crecimiento de los árboles aumenta fuertemente con intervenciones silviculturales y los rendimientos de madera de calidad a futuro pueden estimarse del orden de 10 a 15 veces superiores a los actuales en el bosque natural no manejado.

\section{b) Acciones sugeridas:}

En algunas regiones de Chile, los bosques naturales aún son recursos importantes para desarrollar una actividad forestal sustentable $y$, en otras, el deterioro de los bosques y los problemas ambientales generados son de tal magnitud, que requieren de inversiones que hacen difícil su recuperación sin apoyo estatal.

Las acciones a realizar para lograr una definición de objetivos nacionales para los bosques nativos, implementar un manejo sustentable e incorporarlos al desarrollo forestal del país son:

- Diseñar una política forestal nacional con una definición global de política forestal o una ley marco para el sector, que incluyan una clara definición de las responsabilidades sociales ligadas a la propiedad de los bosques; 
- modificar las normas de manejo para los bosques natiyos, ya que normalmente se persigue sólo la explotación de madera y no existe la intención de manejar el bosque;

- subsidiar el manejo forestal para bosques rentables o no rentables con manejo y la reconversión de actividades agropecuarias en bosques; y otorgar sello de garantía de sustentabilidad para la madera.

La efectividad de las exigencias ambientales internacionales, la seriedad de un sello de estas características para la exportación de productos y las consecuentes ventajas económicas, podrían constituirse en un incentivo decisivo para conservar los bosque y obtener de ellos el máximo beneficio social.

3. El caso de la celulosa frente a las restricciones ambientales. Los requerimientos europeos.

El sello ambiental de la Comunidad Europea (CEE) (o ecoetiquetado) se refiere al uso de sellos con el fin de informar a los consumidores respecto a productos que provocan menores impactos ambientales durante su producción, transporte, almacenamiento y disposición final que otros productos de la misma categoría. Se aplica tanto a los productos de la CEE como a sus importaciones y cumple el rol de instrumento para la promoción de productos provenientes de tecnologías limpias.

Se ha establecido un procedimiento para el otorgamiento del sello, siendo aplicado en primer lugar a las máquinas lavadoras y secadoras y dos productos de la industria papelera, toallas de cocina y papel higiénico. El tema resulta de importancia para Chile, por cuanto permite advertir claramente la relación entre regulaciones ambientales externas y potenciales problemas para la industria de la celulosa, así como para la economía chilena en general.

La industria de la celulosa y el Gobierno deberán enfrentar el problema del sello ambiental de la CEE y desarrollar una estrategia que ayude a ubicar y orientar a la industria de la celulosa en este ambiente nuevo.

Los sellos ecológicos son distintivos que se utilizan con el fin de orientar a los consumidores respecto del impacto en el medio ambiente generado por la producción, uso y desecho de los productos. Sin embargo, surge un gran número de problemas de la comparación de diversos y complejos aspectos del medio ambiente que son representados por un único indicador, cuestión que merece ser debatida. 
El criterio utilizado respecto a los recursos renovables parece tener como objetivo el estímulo al reciclaje de fibra a fin de disminuir la presión sobre los bosques. Este objetivo responde a la realidad forestal europea, que tiene una historia de uso del suelo y de fuentes de recurso fibroso muy distinta a la de los países latinoamericanos y representaría una especie de castigo para la realidad forestal nacional.

El tema de los recursos no renovables, del díxído de carbono, de los orgánicos clorados y del dióxido de azufre empleados en mayor o menor medida en la producción de toallas de papel y papel higiénico, es materia de gran controversia. Esta situación, es particularmente sensible para las exportaciones de pulpa y papel hacia el mercado europeo.

En un escenario de competencia, el sello ecológico puede tener un efecto muy desestabilizador en la economía chilena con el pretexto de protección al medio ambiente. El sello no debería interferir en el comercio libre de los países.

El caso del ecoetiquetado que enfrentan las exportaciones chilenas en Europa ilustra que el proceso de información y reacción frente al tema no siempre ha funcionado debidamente. En este punto se concluye acerca de la importancia de contar con una infraestructura en el sector público ágil y prontamente informada, que permita reaccionar junto al sector exportador de manera oportuna y adecuada. Se necesitaría, asimismo, un apoyo en las negociaciones con las autoridades responsables de la CEE y, se podria trabajar hacia el establecimiento de una infraestructura básica en el ámbito comunitario que opere como contraparte y coordine para terceros países, el ecoetiquetado.

\section{El sector energético. Un caso de estímulo al ahorro por mecanismos de precios.}

La relación entre el consumo de energia y los temas centrales del desarrollo económico, sitúan en una perspectiva apropiada el objetivo del mejoramiento de la calidad ambiental como un objetivo propio del desarrollo. Se señala al desarrollo económico como el principal causante directo o indirecto del deterioro en la calidad ambiental.

El objetivo del desarrollo es el mejoramiento de la calidad de vida, lo que incluye un aumento en la calidad ambiental, por lo que éste es también un objetivo del desarrollo. El problema de la aparente incompatibilidad entre desarrollo y medio ambiente se genera precisamente por el desconocimiento u olvido de esta identidad de objetivos. 
Por otra parte, a pesar del conocimiento real que existe sobre el daño que el deterioro del medio ambiente causa a la sociedad, sus costos han sido generalmente ignorados. La altemativas de desarrollo son en general más baratas, en el corto plazo, cuando no se incorporan restricciones ambientales.

EI problema de fondo radica en lograr crear mecanismos que hagan que se incluyan las variables relevantes ambientales en el proceso normal de toma de decisiones. Se está frente a un típico problema de asignación de recursos escasos -medio ambiente de una calidad determinada-necesarios para fines múltiples.

En el ámbito de estos estudios, se ha postulado que el problema en la asignación adecuada de los recursos ambientales radica en el desconocimiento del precio de mercado de estos recursos. La no existencia de precios, producto principalmente de la indefinición de derechos de propiedad, produciría una sobreexplotación de los recursos con las consecuentes externalidades negativas para algún sector.

Los propios incentivos del mercado actuarian sobre la decisión particular en materia ambiental, llevando a considerar el rol de los precios de mercado y la asignación de derechos de propiedad como elementos fundamentales para que se promueva esta preocupación.

Más concretamente, se produce una asociación entre la idea de contaminación con el consumo de energía, ya que los procesos de combustión traen consigo emisiones importantes de contaminantes. En este sentido, se postula que el aumento en la eficiencia del uso de los energéticos, producto de una mejor combustión, trae como consecuencia directa un ahorro en su consumo y una menor emisión de contaminantes.

El caso chileno permite evaluar estas hipótesis, ya que a partir de mediados de la década de los 70 , se comenzó a aplicar una política energética tendiente a eliminar las distorsiones provenientes de diversos subsidios y fijaciones arbitrarias de precios, con el objeto que éstos reflejasen los verdaderos costos de oportunidad de lọ distintos energéticos. Así, se traspasó al consumidor la decisión de qué, cómo y cuánto de cada energético consumir, basando esta decisión en los verdaderos costos de cada energético. Produjo el doble beneficio de disminuir las emisiones y producir un crecimiento económico más rápido. La aplicación de esta política energética trajo como consecuencia una disminución de $11 \%$ en el consumo de energía final por unidad del PGB, en el período 1975-1990, produciéndose además ahorros mayores en el caso del consumo de petróleo y derivados como el carbón y la electricidad. 
El consumo nacional de petróleo crudo descendió en un 20\%. Esto se debió a un ahorro real de energía como a un efecto de sustitución por otros energéticos más baratos. En el caso del cobre, se ahorró un 17,5\% en la cantidad de energía consumida por tonelada de cobre producida. En el transporte terrestre aumentó la eficiencia energética, producto de la incorporación al país de vehículos con tecnologías más eficientes y menos contaminantes.

En estos ahorros energéticos han jugado un rol importante los consumidores, en el marco de una política de precios realista. Por otra parte, la promoción de políticas sectoriales basadas en el correcto funcionamiento de los mercados, elemento que está presente en las principales economías que consumen las exportaciones chilenas, es un instrumento válido para considerar.

\section{Asentamientos urbanos y su relación con conceptos de desarrollo expuestos en este Informe. Reordenamiento y desarrollo.}

En lo relacionado directamente con el ámbito urbano, al Ministerio de Vivienda y Urbanismo le competen las normas sobre el uso del suelo urbano; al de Transporte, las normas sobre contaminación por fuentes móviles; al de Obras Públicas, normas sobre uso del agua; al de Salud, saneamiento e higiene ambiental.

En lo referente a las actividades vinculadas a los asentamientos urbanos, se establece que los proyectos de desarrollo urbano, los planos reguladores comunales, las propuestas inmobiliarias e industriales y la ejecución de obras de saneamiento ambiental, entre otras, requerirán de estudios de impacto ambiental.

La relación de cada asentamiento con el ambiente natural es muy estrecha, abarcando desde su misma sustentación geográfica y la provisión de insumos básicos, a la posibilidad de desarrollar actividades económicas primarias.

Existen tres grandes categorías de asentamientos humanos: los asentamientos rurales, los urbanos de tipo intermedio y los metropolitanos. En el primer caso, la modernización del sistema agrario ha permitido una relocalización geográfica que se traduce en una urbanización del campo. El auge agro-silvo-pesquero-minero ha hecho aumentar el tamaño y la densidad demográfica de los asentamientos intermedios, que han pasado a ser proveedores de las demandas provenientes del medio rural. 


\section{I.E.I. / Informe sobre comercio internacional, ...}

Las grandes ciudades presentan una disminución relativa de la tasa de crecimiento al emerger sistemas menores centralizados.

Se pueden identificar problemas ambientales de los asentamientos humanos en diferentes localizaciones del territorio, que responden en alguna medida a procesos asociados directa o indirectamente al modelo exportador que se ha venido aplicando, como por ejemplo, contaminación atmosférica por procesos mineros en la II, III y IV Región; contaminación atmosférica por procesos pesqueros en la I, II, III, IV y VIII Región; contaminación derivada de las plantas de celulosa en las VII y VIII Región.

En términos porcentuales, del total de los problemas ambientales regionales tanto del medio natural como construido, un $60,44 \%$ corresponde a los que tienen un impacto directo sobre el medio urbano, representando éstos, en relación a los seleccionados como de mayor relevancia en todo el pais, un $75,91 \%$. Con respecto a la posibilidad de controlar localmente los problemas ambientales urbanos, se observa que más del $50 \%$ de ellos son posibles de intervenir a dicho nivel, bajando este porcentaje en relación al total de los problemas regionales. Ello confirma la fuerte incidencia que en forma creciente está adquiriendo en Chile la acción de los diversos agentes sociales en el ámbito de la comuna y la gestión del municipio en el mejoramiento de la calidad ambiental.

Se propone el desarrollo, perfeccionamiento y aplicación de un conjunto de instrumentos de política, tales como:

- Planes de ordenamiento territorial, como una de las estrategias fundamentales para alcanzar el desarrollo sustentable;

- planes de ordenamiento de usos de suelo;

- orientación del comportamiento de productores, consumidores e inversionistas, en aquellas situaciones donde, según se ha detectado, los comportamientos "naturales" conducen a respuestas distorsionadas y por lo tanto insuficientes; $y$,

- perfeccionamiento del Estado y la gestión urbana, la que se debe dar dentro de un marco de participación y formación de consensos, única manera de canalizar la energía social necesaria, y mediante el soporte de un aparato estatal moderno y capacitado para desarrollar funciones dentro de una complejidad sin precedentes. 